\title{
Controle de anaplasmose bovina através de imunização com Anaplasma centrale ${ }^{1}$
}

\author{
André G.C. Dalto ${ }^{2 *}$, José Reck Junior ${ }^{3}$, João Ricardo Martins ${ }^{3}$, Ana P.G. Bitencourt ${ }^{4}$, \\ Cristiano Feltrin ${ }^{5}$ e David Driemeier ${ }^{4}$
}

\begin{abstract}
Dalto A.G.C., Reck Junior J., Martins J.R., Bitencourt A.P.G., Feltrin C. \& Driemeier D. 2018. [Bovine anaplasmosis control through immunization with Anaplasma centrale.] Controle de anaplasmose bovina através de imunização com Anaplasma centrale. Pesquisa Veterinária Brasileira 38(6):1064-1067. Setor de Grandes Ruminantes, Universidade Federal do Rio Grande do Sul, Av. Bento Gonçalves 9090, Agronomia, Porto Alegre, RS 91540-000, Brazil. E-mail: andre.dalto@ufrgs.br

Bovine anaplasmosis is a major cause of production losses and deaths in Rio Grande do Sul cattle herds. Anaplasma sp. is the main causative agent of cattle disease. It causes hyperthermia, anemia, prostration, abortions and reduces milk production in affected animals. In order to control this hemoparasite on a dairy farm located in the municipality of Eldorado do Sul in Rio Grande do Sul, where the disease incidence was high, 471 animals were immunized with Anaplasma centrale in the search for cross-protectiv immunity for Anaplasma marginale. The property anaplasmosis incidence, which usually was above $30 \%$, became $5 \%$ after the immunization. However, abortions were observed resulting from innoculaition, especially in animals that had less than 90 days of pregnancy. The global number of deaths on the farm dropped considerably given that the main cause of death was the bovine anaplasmosis. $15 \%$ of animals inoculated with $A$. centrale showed clinical symptoms of the disease between 15 and 30 days after immunization and had to be treated with oxytetracycline. The amount of money spent with anaplasmosis treatment decay $85 \%$ after the immunization, which caused significant economic impact on the property.
\end{abstract}

INDEX TERMS: Anaplasmosis, immunization, Anaplasma centrale, Anaplasma marginale, cattle, parasitoses.

RESUMO.- A anaplasmose bovina é uma das principais causas de perdas produtivas e mortes no Rio Grande do Sul em rebanhos bovinos. O Anaplasma marginale é o principal agente causador da enfermidade e provoca hipertermia, anemia, prostração, abortos e perdas produtivas nos bovinos acometidos. Tendo em vista o controle deste hemoparasita em uma propriedade leiteira localizada no município de Eldorado do Sul no Rio Grande do Sul, na qual a incidência da doença era alta, 471 animais foram imunizados com

\footnotetext{
${ }^{1}$ Recebido em 17 de maio de 2017.

Aceito para publicação em 1 de junho de 2017.

${ }^{2}$ Setor de Medicina de Grandes Ruminantes, Universidade Federal do Rio Grande do Sul (UFRGS), Av. Bento Gonçalves 9090, Agronomia, Porto Alegre, RS 91540-000, Brasil. *Autor para correspondência: andre.dalto@ufrgs.br

${ }^{3}$ Departamento de Parasitologia, Instituto de Pesquisas Veterinárias Desidério Finamor (IPVDF), Estrada do Conde 6000, Sans Souci, RS 92990-000, Brasil.

${ }^{4}$ Setor de Patologia Veterinária, Universidade Federal do Rio Grande do Sul (UFRGS), Av. Bento Gonçalves 9090, Agronomia, Porto Alegre, RS 91540-000.

${ }^{5}$ Centro Universitário Ritter dos Reis, Av. Manoel Elias 2001, Passo das Pedras, Porto Alegre, RS 91240-261.
}

Anaplasma centrale na busca de desenvolvimento cruzado para Anaplasma marginale. No experimento foi verificado que a incidência que normalmente era acima de $30 \%$ na propriedade passou para níveis inferiores a 5\%. No entanto, foram verificados abortos decorrentes da imunização, principalmente nos animais que possuíam menos de 90 dias de prenhes. Já o número de mortes globais na fazenda caiu consideravelmente tendo em vista que a principal causa de morte era a anaplasmose bovina. Dos animais inoculados com A. centrale em torno de $15 \%$ apresentaram sintomatologia clínica da enfermidade e precisaram ser tratados com oxitetraciclina no período entre 15 e 30 dias após a imunização. 0 custo com tratamento empregado na propriedade posterior à imunização caiu em torno de $85 \%$ o que provocou impacto significativo economicamente na propriedade.

TERMOS DE INDEXAÇÃO: Anaplasmose, imunização, Anaplasma centrale, Anaplasma marginale, bovinos, parasitoses. 


\section{INTRODUÇÃO}

As principais hemoparasitoses bovinas são Anaplasmose e Babesiose que juntas formam o complexo Tristeza Parasitária Bovina e provocam grande impacto econômico na produção bovina mundial. Seus principais transmissores são vetores biológicos como os carrapatos da Familia Ixodidae e as moscas da Família Tabanidae. Embora sua transmissão também possa se dar de forma mecânica (Kocan et al. 2003, Farias 2007).

A anaplasmose é uma enfermidade hemoparasitária infecciosa que afeta bovinos, ovinos e animais silvestres. Seu principal agente causador é o Anaplasma marginale que é uma Riquétsia (Kocan et al. 2003). Na maior parte do Brasil, onde a população de vetores está presente durante o ano inteiro, os animais são relativamente resistentes à doença, desenvolvendo imunidade nos primeiros meses de vida, devido à infecção precoce pelo $A$. marginale, quando ainda estão protegidos pelos anticorpos colostrais. Nestas regiões, surtos e altas taxas de mortalidade são raros, criando uma condição de estabilidade enzoótica (Souza et al. 2000). Porém, levantamentos epidemiológicos realizados no Brasil têm observado a ocorrência de surtos e casos clínicos de anaplasmose, caracterizando a ocorrência de áreas endêmicas (Ribeiro \& Reis 1981, Araújo et al. 1998). Os sinais clínicos característicos da doença são hipertermia, anemia, debilidade, prostração, icterícia e inapetência (Gonçalves 2000).

Este trabalho teve por objetivo avaliar o impacto produtivo e econômico causado pela imunização com A. centrale.

\section{MATERIAL E MÉTODOS}

Local. Propriedade destinada à produção leiteira localizada no município de Eldorado do Sul no Rio Grande do Sul que possuía bovinos de leite distribuídos entre a raça Holandesa e Jersey.

Amostra. 471 animais que possuíam idade acima de 3 meses de idade.

Protocolo experimental. A cepa de Anaplasma centrale foi cedida pelo Setor de Parasitologia do Instituto de Pesquisas Veterinária Desidério Finamor (IPVDF). A inoculação ocorreu em três etapas distintas para que o período de desenvolvimento de reações fosse observado com maior facilidade e sem afetar o manejo da propriedade. A primeira inoculação ocorreu em maio de 2013, a segunda inoculação ocorreu em junho de 2013 e a última inoculação ocorreu em dezembro de 2013.

Após a imunização foi realizado exame físico nos animais diariamente no período de 15 a 30 dias após a inoculação, para avaliação de possíveis reações a inoculação.

Animais que apresentaram sintomatologia clínica de anaplasmose foram tratados com Oxitetraciclina na dose de $20 \mathrm{mg} / \mathrm{kg}$ em dose única.

Para avaliação das perdas gestacionais foram realizados exames ginecológicos no dia anterior as imunizações e 60 dias após as inoculações do A. centrale.
Para avaliação do número de óbitos foi realizado um levantamento das mortes e suas causas no período correspondente a janeiro de 2010 até dezembro de 2014.

Para avaliação de perdas econômicas foi realizado levantamento do número de animais tratados para anaplasmose clínica no período entre 2000 e 2015.

O Quadro 1 apresenta a distribuição de idade dos animais imunizados e a suas situações gestacionais.

\section{RESULTADOS E DISCUSSÃO}

Dos 471 animais imunizados 57 apresentaram reações após as inoculações de Anaplasma centrale e o Quadro 2 mostra a distribuição das reações conforme a idade. Dentre os animais imunizados alguns apresentaram abortos conforme demonstrado no Quadro 3.

No Quadro 4 demonstra levantamento de prevalência entre 2000 e 2009 (Cruz et al. 2011) e complementado com os dados encontrados na granja entre os anos de 2010 e 2014.

No Quadro 5 está evidenciado o levantamento das mortes ocorridas na propriedade nos anos de 2011, 2012, 2013 e 2014.

Uma correlação entre a utilização de medicamentos para tratamento de animais infectados com anaplasmose entre 2010 e 2014 e os seus custos estão indicados no Quadro 6.

Quadro 1. Número de bovinos imunizados de acordo com idade, gênero e situação gestacional

\begin{tabular}{ccc}
\hline Idade (meses) & Prenhe & Vazia \\
\hline $0-12$ & 0 & 54 \\
$13-24$ & 32 & 12 \\
$25-36$ & 65 & 14 \\
+36 & 126 & 168 \\
TOTAL & 223 & 248
\end{tabular}

Quadro 2. Bovinos imunizados contra Anaplasma sp. que apresentaram reação de 15-30 dias após inoculação conforme idade

\begin{tabular}{cc}
\hline Idade & Fêmea \\
\hline $0-12$ & $8 / 54$ \\
& $(14,8 \%)$ \\
$13-24$ & $6 / 44$ \\
& $(13,6 \%)$ \\
$25-36$ & $13 / 79$ \\
& $(16,4 \%)$ \\
+ de36 meses & $30 / 294$ \\
& $(10,2 \%)$ \\
TOTAL & $57 / 471$ \\
& $(12,1 \%)$
\end{tabular}

Quadro 3. Abortos ocorridos após a inoculação, de acordo com a idade de bovinos fêmea e tempo gestacional (em dias) no momento da imunização contra Anaplasma sp.

\begin{tabular}{cccccc}
\hline Idade (meses) & Abortos 30-60 dias & Abortos 60-90dias & Abortos 90-120dias & Abortos +120dias & Abortos totais \\
\hline $13-24$ & $1(4)$ & $2(5)$ & $1(11)$ & $0(12)$ & $4(32)-12,5 \%$ \\
$25-36$ & $5(10)$ & $2(8)$ & $0(23)$ & $4(24)$ & $7(65)-10,8 \%$ \\
+36 & $16(35)$ & $9(27)$ & $5(29)$ & $1(35)$ & $30(126)-23,8 \%$ \\
TOTAL & $22(49)$ & $13(40)$ & $5(71)$ & $41(223)-18,4 \%$
\end{tabular}


Quadro 4. Porcentagem de bovinos que apresentaram sinais clínicos de anaplasmose e necessitaram de tratamento ao longo dos anos em relação ao rebanho total

\begin{tabular}{cc}
\hline Ano & $\begin{array}{c}\text { \% de animais tratados para } \\
\text { anaplasmose }\end{array}$ \\
\hline 2000 & 43,7 \\
2001 & 32,9 \\
2002 & 36,8 \\
2003 & 33,5 \\
2004 & 33,5 \\
2005 & 44,4 \\
2006 & 38,7 \\
2007 & 9,9 \\
2008 & 11,1 \\
2009 & 13,5 \\
2010 & 40,8 \\
2011 & 38,7 \\
2012 & 39,1 \\
2013 & 37,2 \\
2014 & 4,8
\end{tabular}

Adaptado de Cruz et al. (2011).

Quadro 5. Número de bovinos mortos na propriedade de acordo com o ano

\begin{tabular}{lcccc}
\hline \multicolumn{1}{c}{ Ano } & 2011 & 2012 & 2013 & 2014 \\
\hline Total de mortes & 43 & 41 & 48 & 23 \\
Morte por anaplasmose & 27 & 22 & 28 & 2 \\
& $(62,8 \%)$ & $(53,7 \%)$ & $(58,3 \%)$ & $(8,7 \%)$
\end{tabular}

Quadro 6. Estimativa da utilização de medicamentos para tratamento de anaplasmose em bovinos de acordo com o ano

\begin{tabular}{lccccc}
\hline \multicolumn{1}{c}{ Ano } & 2010 & 2011 & 2012 & 2013 & 2014 \\
\hline Número de animais tratados & 184 & 177 & 181 & 175 & 23 \\
Estimativa de Oxitetraciclina & 9200 & 8850 & 9050 & 8750 & 1150 \\
$\begin{array}{l}\text { (200mg) utilizada em ml } \\
\text { Custo estimado em reais }\end{array}$ & 2.392 & 2.301 & 2.353 & 2.275 & 299
\end{tabular}

(considerando o preço atual de

$50 \mathrm{ml}=\mathrm{R} \$ 13$ reais)

As perdas gestacionais que ocorreram ao longo de 2012 foram de 5,8\%, já em 2013 foi de 12,2\% e 2014 de 5,2\%.

Anaplasmose bovina é uma das principais causas de mortes no Rio Grande do Sul (Farias 2007, Lucena et al. 2010) e por consequência uma das principais causas de perdas econômicas aos criadores de bovinos. Tendo em vista o elevado número de casos dessa afecção foi desenvolvido este trabalho em conjunto com o Setor de Patologia Veterinária da Universidade Federal do Rio Grande do Sul (UFRGS) e o Setor de Parasitologia Veterinária do IPVDF com intuito de alterar esta situação na propriedade em questão.

A inoculação de A. centrale tem como objetivo desenvolver imunidade cruzada contra o A. marginale que é o principal causador da anaplasmose bovina (Martins \& Correa 1995, Souza et al. 2000). Apesar do A. centrale ser menos patogênico que o A. marginale, ele pode desenvolver sinais clínicos da enfermidade como hipertermia, prostração e anemia embora de forma mais branda (Martins \& Correa 1995, Gonçalves 2000).

Animais de diferentes idades foram inoculados com A. centrale e verificou-se que $12,1 \%$ dos animais imunizados tiveram que ser tratados com oxitetraciclina, pois apresentaram hipertermia e prostração. Entretanto, ao ser realizado análise estatística do QI-quadrado, evidenciamos que não houve diferença significativa entre as idades imunizadas. Após o tratamento estes animais apresentaram melhora clínica. Algumas vacinas comerciais compostas por $A$. centrale recomendam aplicação de subdoses de oxitetraciclina no período de reação vacinal para evitar morte dos animais vacinados e garantir imunidade ao animal (Gonçalves 2000, Souza et al. 2000). Entretanto a subdose do antimicrobiano pode selecionar linhagens resistentes de Anaplasma sp. (Kocan et al. 2003). Por esta razão, no presente estudo, somente foram tratados os animais que apresentaram sinais mais acentuados da enfermidade. Não houve grande variação de reação nas diferentes faixas etárias, nas quais entre 10 e $15 \%$ dos bovinos receberam tratamento.

Perdas gestacionais foram detectadas na palpação retal realizada 60 dias após a inoculação de $A$. centrale, a qual apresentou o resultado de 18,4\% abortos. Aplicando-se o teste qui-quadrado nesses dados, obtém-se o valor de 46,5. Esse valor permite afirmar, mesmo a um nível de significância de 99,9\%, que as diferenças observadas são estatisticamente diferentes de zero e que há, portanto, associação entre as variáveis - no caso, entre o número de abortos e a idade gestacional. Historicamente os abortos variam entre 4,5 e $6 \%$ na propriedade, já a média de perdas gestacionais no ano de 2013 foi de $12,2 \%$ e foram superiores as observadas nos anos de 2012 e 2014. Esse aumento pode ser atribuído ao efeito da imunização com Anaplasma centrale. Alguns autores descrevem que essas perdas podem ocorrer devido à hipertermia (Pipano et al. 1985, Meléndez et al. 2003) e outros defendem que as perdas podem ocorrer por transmissão transplacentária (Correa et al. 1978) do agente.

Neste trabalho foram evidenciadas perdas gestacionais entre 10 e $20 \%$ em animais de diferentes idades, porém ficou evidente que a maior parte das perdas gestacionais ocorreu em animais com menos de 90 dias de prenhez.

Entre 2008 e 2014 diversos animais do rebanho foram tratados para anaplasmose e após a imunização a prevalência da enfermidade caiu de maneira acentuada o que evidencia a eficiência da inoculação de Anaplasma centrale. Pode ser observado também que nos anos de 2007, 2008 e 2009 a prevalência da enfermidade esteve a baixo da média do período e isso se deve a um acentuado controle de ectoparasitas que foi realizado na propriedade nesses anos especificamente (Cruz et al. 2011). No entanto, o controle através da imunização obteve prevalência inferior à encontrada nesse período, o que indica a eficiência do procedimento.

Como a principal causa de mortes na propriedade era a anaplasmose, após o controle pela imunização com Anaplasma centrale o número total de perdas de animais também diminuiu consideravelmente na propriedade, o que provocou ganhos econômicos consideráveis para a propriedade devido à diminuição das mortes.

Pela mesma premissa relativa ao controle de anaplasmose, o número de animais que apresentaram a enfermidade e por consequência necessitaram tratamento reduziu como 
apresentado e esse fato representou na propriedade em questão uma economia de $85,6 \%$ nos gastos com oxitetraciclina para tratamento de animais doentes.

Para realizar uma análise mais detalhada do real impacto econômico causado pela anaplasmose nessa propriedade devem ser adicionados os custos com outros medicamentos que visem suprir as necessidades para sobrevivência do animal, os custos de transfusões de sangue, a baixa na produção de leite, as mortes, as mortes embrionárias, as mortes fetais, além do custo com o atendimento veterinário, porem com os dados já obtidos fica claro que houve um forte impacto positivo economicamente aumentando assim a produtividade da fazenda.

\section{CONCLUSÕES}

Bovinos imunizados com Anaplasma centrale desenvolvem imunidade cruzada com Anaplasma marginale, porém a imunização pode causar o desenvolvimento de sinais clínicos e abortos.

A imunização em vacas prenhes na fase inicial de gestação deve ser tratada com atenção, pois foi observado taxa de aborto acima da media do rebanho.

Os resultados da imunização foram positivos pelo ponto de vista econômico, pois após o período de reação vacinal os bovinos imunizados apresentaram prevalência de anaplasmose significativamente inferior aos anos anteriores o que diminuiu drasticamente os custos com medicamentos e com atendimento veterinário.

\section{REFERÊNCIAS}

Araújo F.R., Madruga C.R., Leal C.R.B., Bastos P.A.S. \& Marques A.P.C. 1998. Frequência de anticorpos anti-Anaplasma marginale em rebanhos leiteiros da Bahia. Arq. Bras. Med. Vet. Zootec. 50(3):243-246.

Correa W.M., Correa C.N.M. \& Gottschalk A.F. 1978. Bovine abortion associated with Anaplasma marginale. Can. J. Comp. Med. 42(2):227-228. PMid:667708.
Cruz C.E.F., Raymundo D.L., Cerva C., Pavarini S.P., Dalto A.G.C., Corbellini L.G. \& Driemeier D. 2011. Records of performance and sanitary status from a dairy cattle herd in Southern Brazil. Pesq. Vet. Bras. 31(1):1-9. http:// dx.doi.org/10.1590/S0100-736X2011000100001.

Farias N.A. 2007. Tristeza parasitária bovina, p.524-532. In: Riet-Correa F., Schild A.L., Lemos R.A.A. \& Borges J.R.J. (Eds), Doenças de Ruminantes e Equídeos. Vol.1. Palloti, Santa Maria.

Gonçalves P.M. 2000. Epidemiologia e controle da Tristeza Parasitária Bovina na região do sudeste do Brasil. Ciência Rural 30(1):187-194. http://dx.doi. org/10.1590/S0103-84782000000100030.

Kocan K.M., de la Fuente J., Guglielmone A.A. \& Melendez R.D. 2003. Antigens and alternatives for control of Anaplasma marginale infection in cattle. Clin. Microbiol. Rev. 16(4):698-712. http://dx.doi.org/10.1128/CMR.16.4.698712.2003. PMid:14557295.

Lucena R.B., Pierezan F., Kommers G.D., Yrigoyen L.F., Fighera R.A. \& Barros C.S.L. 2010. Doenças de bovinos no sul do Brasil: 6706 casos. Pesq. Vet. Bras. 30(5):428-434. http://dx.doi.org/10.1590/S0100-736X2010000500010.

Martins J.R. \& Corrêa B.L. 1995. Babesiose e anaplasmose bovina: aspectos destas enfermidades. Pesq. Agropec. Bras. 1(1):51-58.

Meléndez R.D., Toro Benítez M., Niccita G., Moreno J., Puzzar S. \& Morales J. 2003. Humoral immune response and hematologic evaluation of pregnant Jersey cows after vaccination with Anaplasma centrale. Vet. Microbiol. 94(4):335-339. http://dx.doi.org/10.1016/S0378-1135(03)00128-7. PMid:12829387.

Pipano E., Mayer E. \& Frank M. 1985. Comparative response of friesian milking cows and calves to Anaplasma central vaccine. Brit. Vet. J. 1:141-174.

Ribeiro M.F.B. \& Reis R. 1981. Exposição natural de bezerros, em área endêmica de Anaplasma marginale em Minas Gerais. Arq. Esc. Sup. Vet. UFMG 33:63-66.

Souza J.P.C., Soares C.O., Massard C.L., Scofield A. \& Fonseca A.H. 2000. Soroprevalência de Anaplasma marginale em bovinos na mesorregião Norte Fluminense. Pesq. Vet. Bras. 20(3):97-101. http://dx.doi.org/10.1590/ S0100-736X2000000300002. 